\title{
Sexual behaviour: related adverse health burden in the United States
}

\author{
S H Ebrahim, M T McKenna, J S Marks
}

Sex Transm Infect 2005;81:38-40. doi: 10.1136/sti.2003.008300

As part of an analysis of the burden of disease and injury in the United States, we identified and quantified the incidence of adverse health events, deaths, and disability adjusted life years (DALY) attributed to sexual behaviour. In 1998, about 20 million such events (7532/100 000 people) and 29782 such deaths (1.3\% of all US deaths) occurred, contributing to 2161417 DALYs (6.2\% of all US DALYs). The majority of incident health events (62\%) and DALYs $(57 \%)$ related to sexual behaviour were among females, and curable infections and their sequelae contributed to over half of these. Viral infections and their sequelae accounted for nearly all sexual behaviour related deaths-mostly HIV/AIDS. Sexual behaviour attributed DALYs in the United States are threefold higher than that in overall established market economies.

$\mathrm{T}$ he need to address root causes of health issues-rather than a disease, pathology, or organ system-is well recognised. ${ }^{1}$ An assessment of 1990 deaths in the United States concluded that half of all deaths were attributable to nine risk factors, including sexual behaviour (which accounted for 30000 deaths). ${ }^{1}$ Sexual behaviour leads to a variety of harmful consequences, such as unintended pregnancy, social stigma, infections, and chronic psychological or pathological sequelae. ${ }^{2}$

Measuring adverse outcomes of reproduction or sexual behaviour by counting deaths or diseases alone is inadequate for a proper understanding of the dimensions of the issue. Many such adverse outcomes occur at a young age leading to a large component of lifespan lived with disability. Many deaths from such adverse outcomes occur at a young age and such loss of productive life is not captured by mortality statistics. In this first attempt to generate comprehensive summary statistics on the public health burden related to sexual behaviour in the United States, we present estimates of incidence of adverse health events (morbidity and their sequelae), mortality; and disability adjusted life years (DALYs). DALY is a time based, composite indicator of the burden of disease that adds loss of life years caused by premature death and loss of healthy life as a result of morbidity and associated disability. ${ }^{23}$

\section{METHODS}

A simple comprehensive approach for identifying adverse health outcomes of root causes such as sexual behaviour is not available. Many adverse health outcomes for which sexual behaviour have a role have multifactorial aetiology. For example, only a proportion of hepatitis B virus (HBV) is transmitted sexually, and only a proportion of liver cancer is caused by HBV. Sorting out the relative contribution of sexual behaviour for some conditions and quantification of such conditions require disparate approaches and varying data sources. Similarly, calculation of DALYs for a given condition requires information on the natural history of that condition including incidence and duration of illness, the extent of disability, and the number of deaths, some of which are not readily available. For this analysis, we adapted and updated the methodology used in the global burden of disease study initiated by the World Bank and the World Health Organization, and other attempts to assess various components of sexual behaviour related health burden. ${ }^{2-5} \mathrm{We}$ used the data compiled for the US Burden of Disease Study including published estimates of incidence of sexually transmitted diseases (STD) and reproductive morbidity and mortality, national vital statistics, notifiable disease surveillance system, national surveys, outpatient and hospital discharge summaries, prevalence data from individuals attending healthcare services, and expert opinion..$^{3-12}$ Base year for the US Burden of Disease study was 1996.

We included $100 \%$ of all major sexually transmitted infections. For conditions with risk factors other than sexual behaviour (for example, HIV), or aetiology (for example, liver disease), we identified the sexual behaviour attributable fraction from the literature (table 1). ${ }^{24-68}$ Consistent with assumptions in the report of the World Health Organization, maternal and infant conditions related to unintended pregnancy and all elective abortions that were not medically indicated were considered as adverse outcomes of sexual behaviour. ${ }^{2}$ Sexual behaviour related conditions with insufficient data on attributable fractions (for example, bacterial vaginosis, rape associated violence and post-traumatic stress disorders) were excluded.

\section{RESULTS}

Overall, in the United States, in 1998, about 20 million adverse health events (7532 per 100000 people) and 29745 deaths (1.3\% of US deaths) were attributed to sexual behaviour, contributing to 2161417 DALYs $(6.2 \%$ of US DALYs $\left.^{3}\right)$. Females suffered the majority of incident sexual behaviour related adverse health events (62\%) and DALYs $(57 \%)$, whereas males suffered the majority of deaths $(66 \%)$ (table 1). If HIV related mortality were excluded, more than $80 \%$ of sexual behaviour related mortality would be those among women. Among females, more than half of the incident events and DALYs were contributed by curable infections and their sequelae. However, deaths from curable sexually transmitted infections were rare; the majority of unsafe sex related deaths are from viral infections and their sequelae (men, 99.5\%; women 96.8\%). Cervical cancer and HIV are the leading causes of such mortality among females, whereas HIV was by far the single leading cause among males. Also, viral diseases caused the majority of sexual behaviour attributed health burden among males.

Abbreviations: DALY, disability adjusted life years; HBV, hepatitis B virus; HPV, human papillomavirus; STD, sexually transmitted diseases 
Table 1 Sexual behaviour related adverse health burden, United States, 1998

\begin{tabular}{|c|c|c|c|c|}
\hline & $\begin{array}{l}\text { Attributable to } \\
\text { sex (\%) }\end{array}$ & $\begin{array}{l}\text { Incidence (rounded to } \\
\text { the nearest 100) }\end{array}$ & $\begin{array}{l}\text { Number of } \\
\text { deaths }\end{array}$ & $\begin{array}{l}\text { Disability adjusted } \\
\text { life years (DALY) }\end{array}$ \\
\hline \multicolumn{5}{|c|}{ Female (population: 135474000 ) } \\
\hline \multicolumn{5}{|c|}{ Curable diseases and their sequelae: } \\
\hline Chlamydia & 100 & 2511200 & 0 & 10381 \\
\hline Gonorrhoea & 100 & 321300 & 4 & 1326 \\
\hline Trichomoniasis & 100 & 2600000 & 0 & 10266 \\
\hline Syphilis & 100 & 33100 & 48 & 790 \\
\hline Other curable STDs & 100 & 200 & 0 & 90 \\
\hline PID & 65 & 429300 & 165 & 2136 \\
\hline Infertility & 10 & 598000 & 0 & 843747 \\
\hline \multicolumn{5}{|c|}{ Viral diseases and their sequelae: } \\
\hline Genital herpes & 100 & 586300 & 0 & 9941 \\
\hline HPV & 100 & 3245000 & 0 & 0 \\
\hline Cervical cancer & 100 & $12700+$ & 4921 & 59418 \\
\hline Hepatitis B virus & $33 \ddagger, 58 \S$ & 53300 & 381 & 146387 \\
\hline Hepatitis $C$ virus & $9 \ddagger, 20 \S$ & 3800 & 288 & 10131 \\
\hline HIV & $57 \%$ & 8200 & 4234 & 110019 \\
\hline \multicolumn{5}{|l|}{ Pregnancy related: } \\
\hline Pregnancy outcome* & t† & 615200 & 107 & 20321 \\
\hline Elective abortions* ${ }^{*}$ & $94 \ddagger \ddagger$ & 1298000 & ** & ef \\
\hline Total & & 12315600 & 10148 & 1224953 \\
\hline \multicolumn{5}{|c|}{ Male (population: 129810 000) } \\
\hline \multicolumn{5}{|c|}{ Curable diseases and their sequelae } \\
\hline Chlamydia & 100 & 489200 & 0 & 2026 \\
\hline Gonorrhoea & 100 & 329000 & 2 & 1352 \\
\hline Trichomoniasis & 100 & 2400000 & 0 & 9445 \\
\hline Syphilis & 100 & 37000 & 84 & 1598 \\
\hline Other curable STDs & 100 & 200 & 4 & 120 \\
\hline Urethral stricture & $\S \S$ & 23400 & 0 & 209 \\
\hline $\begin{array}{l}\text { Epididymitis } \\
\end{array}$ & $\S \S$ & 49700 & 0 & 444 \\
\hline Infertility & $\S \S$ & 7500 & 0 & 15336 \\
\hline \multicolumn{5}{|c|}{ Viral diseases and their sequelae: } \\
\hline Genital herpes & 100 & 413700 & 0 & 7014 \\
\hline HPV & 100 & 2255000 & 0 & 0 \\
\hline Hepatitis $B$ virus & $33 \ddagger, 58 \S$ & 1593000 & 711 & 279624 \\
\hline Hepatitis $C$ virus & $9 \ddagger, 20 \S$ & 31700 & 612 & 62275 \\
\hline HIV & 72 & 36800 & 18221 & 557021 \\
\hline Total & & 7666200 & 19634 & 936464 \\
\hline \multicolumn{2}{|c|}{ US total (population: 265284000 ) } & 10091000 & & \\
\hline \multicolumn{5}{|c|}{ 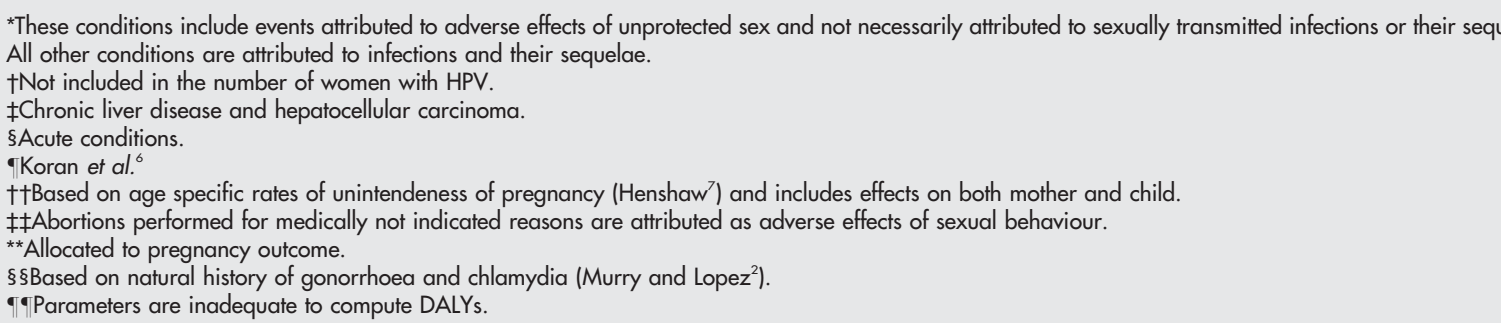 } \\
\hline
\end{tabular}

\section{DISCUSSION}

Sexual behaviour attributed DALYs in the United States are threefold higher than those in overall established market economies. ${ }^{2}$ Our analysis was comprehensive and included more unsafe sex related conditions than were considered in previous analyses for the United States (such as hepatitis, infertility, reproductive sequelae in men, maternal conditions, and abortions). ${ }^{45}$ Even so, our estimates probably reflect the lower bound of the sexual behaviour related public health burden in 1998. For instance, for HIV infection, we included only the percentage attributable to sexual transmission alone, and not in combination with other risk factors such as injecting drug use. If we included people who had other risk factors (that is, in addition to sexual behaviour) for HIV transmission, part of the remaining 27\% HIV/AIDS burden among males and 29\% females would be attributable to sexual transmission. Sexual behaviour attributed DALYs, particularly those attributed to HIV/AIDS, may vary slightly for more recent years because of the declines in HIV/AIDS deaths, increases in the number of people living with HIV/ AIDS, and the resurgence of bacterial STDs and unsafe sex among some population groups. ${ }^{6}$ DALYs may not capture the full social and economic dimensions of reproductive morbidity and those arising from some prevalent viral infectionsfor example, 45 million people in the United States have genital herpes and 20 million carry the human papillomavirus (HPV). ${ }^{2}$

Women bear a disproportionately high proportion of the overall sexual behaviour attributable health burden in the United States. The role of sexual behaviour is generally far removed from discussions and prevention programmes that address cervical cancer. Cervical cancer causes more deaths than HIV and such deaths generally occur among older women. However, HIV affects younger women and therefore the DALY estimates for HIV are nearly twofold higher than cervical cancer. Among women, curable infections and their sequelae still prevail as the leading contributor to disease and disability. Although this analysis was not stratified by age groups, it is clear that adolescents and young women are at the highest risk of complications from unsafe sex (pregnancy, abortions, infections) which can cover an entire lifespan. ${ }^{2} 89$ Despite the availability of contraceptive choice in the United States, $78 \%$ of pregnancies among 15-19 year old women in 1994 were unintended. ${ }^{6}$ Knowledge about HPV and use of the 


\section{Key messages}

- Sex attributed public health burden in the United States is threefold higher than that in other established market economies

- The majority of sex attributed burden in the United States is among women

- Curable infections and their sequelae remain the leading contributors to sexual behaviour related burden among US women

- HIV is the single leading cause of the sex related burden among men in the United States

Papanicolaou smear test for the detection of cervical abnormalities is still low among young women. ${ }^{2}$

Estimation of health burden with respect to a root cause (such as sexual behaviour) is limited by the conceptual difficulties in defining the link between exposure and health event, deficiencies in the primary databases, and by the variable approaches used in various studies to derive attributable fractions. ${ }^{1-35}$ Risk information is recorded hierarchically for some pathogens such as HIV and hepatitis viruses. In such instance, people who had both sexual behavioural risk and injecting drug use risk may be subsumed in the injecting drug use category. Information on risk behaviour or exposure is limited in the medical and surveillance records and no risk is identified for a significant proportion of cases both for HIV and hepatitis viruses. ${ }^{12} 13$ Therefore, the attributable fractions developed for some conditions by themselves may be underestimates. Another concern is the time lag between risk and disease acquisition and disease occurrence. For example, exact timing of hepatitis B virus transmission that resulted in hepatocellular carcinoma may be unknown in most cases. The percentage of hepatitis B virus transmitted sexually varies over time (or unknown for some periods) leading to inconsistency in data used for attributable fractions to compute the proportion of hepatocellular carcinoma that is related to hepatitis B virus. Lastly, period of time lived with disability is variable and influenced by many determinants including access to care, introduction of newer treatment options, and time taken to access such options. Further, reporting delays, misclassification, and incompleteness in reporting of varying levels are inherent limitations of some primary data sources used for counting of deaths and morbidity including cancer and other disease registries, surveillance systems, and vital statistics. ${ }^{12-16}$

It is generally acknowledged that the field of sexual and reproductive health should not only address the key adverse outcomes but pay attention to positive including life enhancing aspects of sexuality. Methods to estimate such benefits and, more so, how to translate such findings to action for programs remain unclear. In the meantime, the quantification attempts, such as this which focused on the adverse outcomes, compel us to further refine the methodology, and the findings offer a mandate that help us focus public health policy on fundamental factors that affect a wide variety of diseases. Despite the many criticisms of the DALY as a measurement unit, ${ }^{15}{ }^{16}$ it represents a major conceptual advance to assess public health burden providing a common measure for fatal and no fatal outcomes.

Our findings highlight the extent and range of adverse effects of sexual behaviour. Such recognition calls for greater emphasis on the design and implementation of sexual risk reduction programmes. Increasing coverage of hepatitis B vaccine will undoubtedly reduce sexually transmitted hepatitis $\mathrm{B}$ virus related burden. However, given the size and chronicity of HIV, HPV, and other hepatitis virus epidemics, the overall health burden related to sexual behaviour is unlikely to decline rapidly in coming years. Interventions among adolescents to delay age at first sexual contact, widespread Papanicolaou testing and use of hepatitis B vaccine, screening and treatment of curable STDs, and correct and consistent use of condoms and contraceptives can reduce the sexual behaviour related public health burden substantially. Nearly all sexual behaviour related adverse health events are preventable.

\section{CONTRIBUTORS}

The overall US Burden of Study (USBoD) was conceived and initiated by JSM, and directed by MTMcK; SHE developed estimates for some components of the USBoD; SHE designed and conducted the analysis on sexual behaviour as a risk factor using the US BoD data and wrote the paper with contributions from JSM and MTM.

\section{Authors' affiliations}

S H Ebrahim, M T McKenna, National Center for HIV/AIDS, STD, TB Prevention, Centers for Disease Control and Prevention, Atlanta, GA, USA

J S Marks, National Center for Chronic Disease Prevention and Health Promotion, Centers for Disease Control and Prevention, Atlanta, GA, USA

Correspondence to: Shahul H Ebrahim, MD, PhD, Centers for Disease Control and Prevention (CDC, Mail Stop E-46), 1600 Clifton Road, Atlanta, GA 3033, USA; sebrahim@cdc.gov

Accepted for publication 17 April 2004

\section{REFERENCES}

1 McGinnis J, Foege WH. Actual causes of death in the United States. JAMA 1993;270:2207-17

2 Murry CJL, Lopez AD, eds. Health dimensions of sex and reproduction: the global burden of sexually transmitted diseases, HIV, maternal conditions, perinatal disorders, and congenital anomalies. Geneva: World Health Organization, 1998.

3 Michaud CM, Murray CJL, Bloom BR. Burden of disease-implications for future research. JAMA 2001;285:535-9.

4 Cates W Jr. Estimates of the incidence and prevalence of sexually transmitted diseases in the United States (review). Sex Transm Dis 1999;26(Suppl 4):S2-7.

5 Ebrahim SH, Peterman TA, Zaidi AA, et al. Mortality related to sexually transmitted diseases in US women, 1973 through 1992. Am J Public Health 1997;87:938-44.

6 Koran JM, Fleming PL, Steketee RW, et al. HIV in the United States at the turn of the century: an epidemic in transition. Am J Public Health 2001;91:1060-8.

7 Henshaw SK. Unintended pregnancy in the United States. Fam Plann Perspect 1998;30:24-9.

8 Wilcox LS, Mosher WD. Use of infertility services in the United States. Obstet Gynecol 1993;82:122-7.

9 Rein DB, Kassler WJ, Irwin KL, et al. Direct medical costs of pelvic inflammatory disease and its sequelae: decreasing, but still substantial. Obstet Gynecol 2000;95:397-402.

10 Torres A, Forrest JD. Why do women have abortions? Fam Plann Perspect 1988;20:169-76.

11 Centers for Disease Control and Prevention. Pregnancy-related deaths among Hispanic, Asian/Pacific Islander, and American Indian/Alaska Native women-United States, 1991-1997. MMWR 2001;50:361-4.

12 Centers for Disease Control and Prevention. Recommendations for prevention and control of hepatitis $C$ virus (HCV) infection and HCV-related chronic disease. MMWR 1998:47:1-39.

13 Klevens RM, Fleming PL, Li J, et al. The completeness, validity, and timeliness of AIDS surveillance data. Ann Epidemol 2001;11:443-9.

14 Wingo PA, Landis S, Parker S, et al. Using cancer registry and vital statistics data to estimate the number of new cases and deaths in the United States for the upcoming year. J Reg Management 1998;25:43-51.

15 AbouZahr C, Vaughan JP. Assessing the burden of sexual and reproductive ill-health: questions regarding the use of disability-adjusted life years. Bull World Health Organ 2000;78:655-65.

16 Williams A. Calculating the global burden of disease: time for a strategic reappraisal? Health Economics 1999;8:1-8. 